\title{
La fissuration d'un sol argileux au cours du des- sèchement. I. Description in situ
}

Vincent HALLAIRE

I.N.R.A., Station de Science du Sol, Centre de Recherches d'Avignon, F84140 Montfavet

RÉSUMÉ

\begin{abstract}
La fissuration d'un sol argileux est étudiée in situ, le dessèchement étant assuré par une prairie. Pour décrire l'évolution du réseau fissural au cours du dessèchement, on estime la taille des éléments structuraux individualisés par les fissures (dont ils sont le « complémentaire » dans le sol) : les mottes et leurs éléments constitutifs, les " peds ». On compare l'évolution de la masse de ces éléments à des mesures densimétriques sur des cylindres de sol desséchés par évaporation. Ces méthodes permettent de distinguer 2 phases de lissuration lors du retrait . le dessèchement provoque d'abord une fragmentation du sol, créant un réseau serré de fissures fines ; puis il provoque une prise en masse, créant un réseau lâche de fissures larges.
\end{abstract}

Mots clés additionnels : Argile, retrait du sol, fissure, densité apparente, mottes, peds, structure du sol.

Cracking of a clayey soil was studied in situ, during drying under grass cover. In order to evaluate the changes of width and of spacing of the cracks during drying, we assessed by gravimetric measurements the size of the complementary structural elements which they isolate in the soil : i.e. the clods and their constitutive elements, the peds. These results were compared with density measurements of soil cores dried by evaporation. These methods distinguished 2 stages of cracking during shrinkage : drying first caused the soil to split up into a dense network of thin cracks; then caused a "sintering", creating a loose network of wide cracks.

Additional key words : Clay, soil shrinkage, crack, bulk density, clods, peds, soil structure.

\section{INTRODUCTION}

Le dessèchement d'un sol riche en argile gonflante provoque une réorganisation de ses constituants ; il en résulte un retrait, se manifestant par une modification de la porosité du sol et notamment par l'apparition de fissures.

Depuis HAINES (1923), les variations densimétriques de ces sols ont été étudiées sur des échantillons remaniés (FrANZMEIER \& ROSS, 1968) ou non (HOLMES, 1955 ; WHITE, 1962 ; FOX, 1964), à des échelles variées (MONNIER et al., 1973 ; BERNDT \& COUGHLAN, 1976 ; STENGEL, 1979 ; YULE \& RITCHIE, $1980 a$ et $b$; BRUAND, 1985) ; elles ont été reliées à leur composition granulométrique (FIĖs \& STENGEL, 1981 $a$ et $b$ ) et minéralogique (TESSIER \& PÉDRO, 1980 ; TESSIER, 1984).

Si ces études ont abouti à une meilleure connaissance des processus mis en cause, la description mor-

(Adresse actuelle: I.N.R.A., Laboratoire de Science du Sol, 65, rue de St-Brieuc, 35042 Rennes.) phologique des fissures qui en résultent reste souvent limitée à des situations minéralogiques particulières : dans des polders (RIJNIERSCE, 1984) ou des vertisols (EDELMAN \& BRINKMAN, 1962 ; DE VOS \& VIRGO, 1969 ; VIRGO, 1981). Dans ce dernier type de sol, la porosité bimodale très marquée (micropores intramassifs et macrofissures) a permis à CABIDOCHE et al. (1986) de repérer et de décrire les fissures par des mesures de résistivité électrique.

Dans cet article, nous étudierons un sol argileux où la continuité et l'étendue du spectre poral ne permettent pas de distinguer des classes de pores aussi tranchées ; nous tenterons une description morphologique des fissures par 2 voies :

- au laboratoire, l'évaluation du volume des fissures s'effectuera par des mesures de la densité d'échantillons non perturbés et desséchés par évaporation ;

- in situ, c'est-à-dire dans un sol où le dessèchement est assuré par une culture, la méthode de description des fissures sera basée sur la mesure de la taille des éléments structuraux (mottes ou «peds») individualisés par ces fissures : dans un sol où celles-ci 
s'organisent en réseau, ces éléments structuraux constituent au sens géométrique le complémentaire des fissures.

Après avoir envisagé les problèmes que posent la mise en évidence et le prélèvement de ces éléments structuraux, nous tenterons de relier les diverses approches pour caractériser l'évolution des réseaux de fissures.

\section{MATÉRIEL ET MÉTHODES}

\section{A. Le sol}

La parcelle d'étude est située aux Vignères (Vaucluse), dans la plaine d'Avignon. Exploitée en prairie (fétuque élevée implantée depuis 6 ans), elle ne reçoit aucune irrigation, afin d'assurer un dessèchement maximal du sol par absorption racinaire au cours de la saison de végétation.

Le sol s'est développé sur des matériaux alluviaux récents riches en argile gonflante (montmorillonite), reposant à $2 \mathrm{~m}$ sur un lit de galets. Cette étude n'a porté que sur une profondeur de $1 \mathrm{~m}$, de façon à rencontrer les variations d'humidité maximales.

Le profil reste très homogène sur cette profondeur : la texture y varie peu, avec une fraction argileuse comprise entre 50 et 55 p. 100 ; la structure est polyédrique anguleuse, avec une tendance cubique très marquée, surtout en profondeur. Malgré l'absence de différenciation nette d'horizons pédologiques, nous distinguerons cependant au sein du profil des couches de sol où l'on pourra considérer l'humidité homogène. En excluant les $10 \mathrm{~cm}$ supérieurs à cause du mat racinaire qui s'y développe, nous étudierons 5 couches d'épaisseur $20 \mathrm{~cm}$ : ces couches seront dénommées A $(10-30 \mathrm{~cm}), \mathrm{B}(30-50 \mathrm{~cm}), \mathrm{C}(50-70 \mathrm{~cm}), \mathrm{D}(70-$ $90 \mathrm{~cm})$ et $\mathrm{E}(90-110 \mathrm{~cm})$.

Le tableau 1 donne la granulométrie de ces 5 couches.

TABLEAU 1

Caractéristiques granulométriques du sol étudié. Textural characteristics of the studied soil.

\begin{tabular}{|c|c|c|c|c|c|c|}
\hline \multirow{2}{*}{$\begin{array}{l}\text { Couche } \\
\text { de sol }\end{array}$} & \multicolumn{5}{|c|}{ Classes granulométriques $(\mu \mathrm{m}) \%$} & \multirow{2}{*}{$\begin{array}{l}\text { Calcaire } \\
\text { total } 0 \%\end{array}$} \\
\hline & $0-2$ & $2-20$ & $20-50$ & $50-200$ & $200-2000$ & \\
\hline A & 51,3 & 30,4 & 7,4 & 8,6 & 2,3 & 27,5 \\
\hline B & 51,5 & 29,6 & 7,3 & 9,1 & 2,5 & 28,2 \\
\hline $\mathrm{C}$ & 53,2 & 31,1 & 6,5 & 7,3 & 1,9 & 29,1 \\
\hline $\mathrm{D}$ & 54,9 & 32,6 & 5,4 & 5,6 & 1,5 & 29,5 \\
\hline $\mathrm{E}$ & 53,2 & 34,9 & 4,5 & 5,6 & 1,8 & 32,3 \\
\hline
\end{tabular}

\section{B. Mesure du volume des fissures sur des monolithes de sol}

Des cylindres de sol non perturbé ont été prélevés à saturation à différentes profondeurs, à l'aide d'anneaux métalliques de volume $1,272 \mathrm{dm}^{3}$ (hauteur $7,2 \mathrm{~cm}$, diamètre $15 \mathrm{~cm}$ ) ; ils ont ensuite été desséchés par évaporation à l'air libre.
Tout au long du dessèchement, on a suivi l'humidité par pesée du cylindre, et son volume par mesure du retrait vertical et du retrait latéral (fig. 1) :

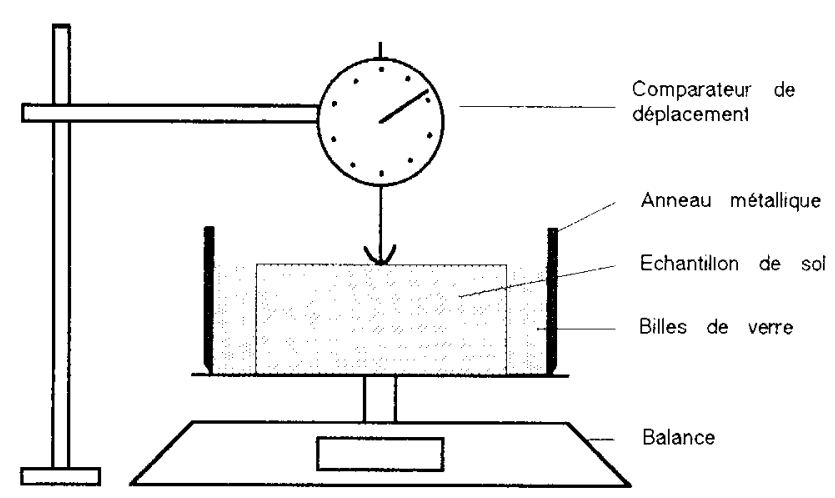

Figure 1

Mesure du retrait vertical (par comparateur de déplacement) et du retrait lateral (par billes de verre) d'un cylindre de sol.

Measurement of vertical shrinkage (with a displacement transducer) and of lateral shrinkage (with glass beads) in a core sample.

- le retrait vertical au moyen d'un comparateur de déplacement (précision $0,01 \mathrm{~mm}$ ) ;

- le retrait latéral au moyen de billes de verre, de diamètre $200 \mu \mathrm{m}$, introduites entre le cylindre de sol et l'anneau métallique : connaissant la densité d'empilement des billes, on calcule le volume qu'elles occupent.

A partir du volume du cylindre, on peut calculer son indice des vides. Par ailleurs, des courbes de retrait ont été effectuées sur des petits agrégats de 2 $3 \mathrm{~mm}$, par mesure de la poussée d'Archimède dans le pétrole, selon la méthode proposée par MONNIER et al. (1973) ; ces courbes permettent de calculer l'indice des vides des agrégats quelle que soit l'humidité.

En désignant comme fissures tous les vides du sol externes aux agrégats, la différence entre l'indice des vides du cylindre et celui des agrégats nous donne l'indice des vides fissuraux. Le calcul de cet indice des vides fissuraux a été explicité par ailleurs (HALLAIRE, 1984).

\section{Caractérisation des mottes et des « peds 》}

\section{Prélèvement des mottes}

Afin de suivre l'évolution au cours du dessèchement de la taille des éléments structuraux individualisés par les fissures, on a prélevé des mottes de terre sur toute une saison de végétation de la fétuque, de façon à couvrir toute la gamme d'humidité in situ. Selon l'intensité de l'évapotranspiration, la fréquence des prélèvements variait de 7 à 15 jours ; entre la quasisaturation du sol (fin mars) et son dessèchement maximum (fin août), 14 prélèvements ont permis de recueillir, à chaque fois, une vingtaine de mottes sur chacune des 5 couches de sol. Au total 1350 mottes ont été prélevées sur tout le profil et durant toute la saison.

L'extraction était effectuée à la main, après observation par l'opérateur des éventuelles solutions de 
continuité séparant ces mottes. Cette opération est d'autant plus aisée que le réseau fissural est visible, encore ne prend-elle pas en compte les fissures plus fines pouvant exister à l'intérieur des mottes ainsi définies. Par contre, lorsque le réseau fissural est inexistant ou trop fin pour être observé, le prélèvement se fait plus arbitraire et la taille de la motte obtenue résulte plus des contraintes mécaniques exercées lors de l'extraction et du transport que d'une réelle individualisation structurale.

\section{Masse et volume des mottes}

Après pesée de la motte, on mesure son volume par poussée d'Archimède dans l'eau : elle est pour cela enrobée de paraffine ou, si son volume est trop important pour permettre cette technique (à partir de $3 \mathrm{dm}^{3}$ environ), enfermée dans un sac plastique étroitement appliqué à la motte par dépression.

Ces données permettent le calcul de la masse volumique humide de la motte.

\section{Individualisation des «peds»}

Le prélèvement de la motte met en évidence les plus larges fissures, que l'on peut détecter à l'œil nu. Pour révéler les éventuelles fissures plus fines subsistant au sein des mottes, on les a débarrassées de leur enrobage et soumises à une chute d'une hauteur standard de $2 \mathrm{~m}$ sur une surface plane. Les éléments issus de la chute sont pesés individuellement.

La fracturation d'une motte au cours d'une chute peut cependant être indépendante d'une structuration interne si sa structure est continue, et ne résulter que d'une contrainte mécanique à l'emplacement du choc. Aussi faudrait-il pouvoir distinguer les éléments structuraux individualisés par la chute, réellement dépendants d'une fissuration interne à la motte, et les « éclats » dus à l'action mécanique mais indépendants de la structure interne.

Dans la pratique, nous admettrons ici que le plus gros des éléments issus de la chute est un indicateur du degré de fissuration interne à la motte, et cu'il suffira à caractériser sa structure interne. Selon la terminologie proposée par BREWER (1964), cet élément structural séparé de ses voisins par des zones de faible résistance ( surfaces of weakness ») correspond au " ped primaire », tandis que la motte dont il est issu correspond au ped secondaire ou tertiaire. Nous retiendrons ici le terme de «ped» pour le désigner et le distinguer de la motte, en gardant toutefois à l'esprit le caractère «mécanique » ayant permis d'obtenir le ped à partir de la motte : le ped défini ici ne peut être retenu qu'à titre indicatif pour estimer l'importance de la fissuration intramotte; il ne saurait se substituer à un élément structural défini selon des critères morphologiques.

Par la suite, le rapport de la masse $M_{p}$ du ped à la masse $M_{m}$ de la motte nous permettra de juger si le ped peut ou non suffire à caractériser l'état structural de la motte.

\section{Humidité}

L'humidité est supposée homogène au sein de la motte, donc identique pour la motte et le ped. Elle est mesurée in fine par séchage à $105^{\circ} \mathrm{C}$ d'une fraction du ped.

Cette donnée permet de convertir la masse volumique de la motte en masse volumique sèche.

\section{RÉSULTATS}

\section{A. Evolution des fissures internes à un monolithe de sol}

La figure 2 représente l'évolution de l'indice des vides fissuraux d'un cylindre de sol prélevé à une profondeur de $40 \mathrm{~cm}$.

$\mathrm{Au}$ cours du dessèchement, le retrait apparaît s'opérer en 2 temps :

- lorsque la teneur en eau pondérale $W$ passe de 30 à 23 p. 100, l'indice des vides fissuraux augmente. Le retrait provoque donc la formation de fissures ;

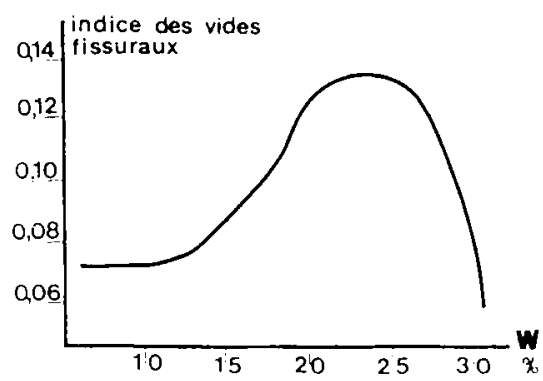

Figure 2

Variation de l'indice des vides fissuraux d'un cylindre de sol avec l'humidité pondérale $W$.

Void ratio of cracks in a core sample versus gravimetric water content $W$.

- pour des teneurs en eau inférieures à 23 p. 100 , l'indice des vides fissuraux diminue progressivement. Il y a disparition, à l'échelle considérée, d'un volume de fissures précédemment ouvertes : nous appellerons ce phénomène "prise en masse ». Dans cette gamme d'humidité, l'augmentation du volume des vides due au retrait ne se situe donc pas dans le monolithe, mais à l'extérieur: ce sont les espaces périphériques au cylindre qui s'accroissent.

La figure 3 illustre ce processus : en $3 a$, le dessèchement débute ( $W=29$ p. 100), les fissures apparaissent ; en $3 b(W=23$ p. 100$)$, leur volume atteint son maximum ; en $3 \mathrm{c}(W=12 \mathrm{p} .100)$, la prise en masse les a refermées, au profit de l'espace annulaire.

\section{B. Evolution de la masse volumique des mottes}

Les mottes ont ici été regroupées par classe d'humidité, chaque classe couvrant une gamme de teneur en eau pondérale de 1 p. 100. Le tableau 2 donne, par couche de sol et par classe d'humidité, la masse volumique sèche moyenne des mottes, $\gamma_{m}$. Des tests statistiques de comparaison de populations (tests de 
Figure 3

Evolution du réseau de fissures à la surface d'un cylindre de sol au cours du dessèchement. a) $W=29$ p. 100 ; b) $W=23$ p. 100 ; c) $W=12 \mathrm{p} .100$

TABLEAU 2

Masse volumique sèche des mottes $\gamma_{m}$ par classe d'humidité et par couche de sol.

Dry bulk density of the clods $\gamma_{m}$ for each class of moisture and for each soil laver.

\begin{tabular}{|c|c|c|c|c|c|}
\hline \multirow{2}{*}{$\begin{array}{c}\text { Teneur en } \\
\text { eau } \\
\text { pondérale } \\
(\%)\end{array}$} & \multicolumn{4}{|c|}{ Couche de sol } & \multirow[b]{2}{*}{$\mathrm{E}$} \\
\hline & A & B & C & $\mathrm{D}$ & \\
\hline 27 & - & - & - & - & 1,457 \\
\hline 26 & - & - & 1,408 & 1,454 & 1,470 \\
\hline 25 & 1,377 & 1,414 & 1,413 & 1,477 & 1,479 \\
\hline 24 & 1,447 & 1,440 & 1,454 & 1,499 & 1,513 \\
\hline 23 & 1,457 & 1,468 & 1,464 & 1,536 & 1,519 \\
\hline 22 & 1,492 & 1,483 & 1,474 & 1,546 & 1,550 \\
\hline 21 & - & 1,511 & 1,558 & 1,558 & 1,531 \\
\hline 20 & 1,569 & 1,541 & 1,570 & 1,594 & 1,596 \\
\hline 19 & 1,588 & 1,589 & 1,576 & 1,612 & 1,614 \\
\hline 18 & 1,608 & 1,570 & 1,622 & 1,655 & 1,653 \\
\hline 17 & 1,618 & 1,609 & 1,647 & 1,677 & 1,685 \\
\hline 16 & 1,620 & 1,647 & 1,622 & 1,684 & 1,728 \\
\hline 15 & 1,669 & 1,666 & 1,662 & - & - \\
\hline 14 & 1,696 & 1,679 & 1,641 & - & - \\
\hline
\end{tabular}

KOLMOGOROV-SMIRNOV) ont montré que, quelle que soit l'humidité, il n'y a pas de différence significative entre les 3 couches supérieures A, B et C, ni entre les 2 couches inférieures $D$ et $E$.

En première approximation, l'augmentation de la masse volumique des mottes au cours du dessèchement semble régulière : le retrait continu des agrégats provoque un retrait continu des mottes.

\section{Evolution de la masse des mottes}

Dans cette phase descriptive de l'étude de la fissuration, nous nous limiterons à suivre l'évolution de la masse humide des éléments structuraux - mottes et peds - , en fonction de leur teneur en eau pondérale ; lors d'une analyse ultérieure, ces données brutes donneront lieu à une interprétation quantitative plus précise.

De même que précédemment, on a pu vérifier que, quelle que soit l'humidité, la distribution de la masse des mottes n'était pas significativement différente entre les couches A, B et C, ni entre les couches D et E. On les a donc regroupées en 2 ensembles : couches supérieures et couches inférieures.
Par ailleurs un test statistique (test de STUDENT) a montré que ces distributions suivaient une loi lognormale ; pour simplifier l'analyse, nous prendrons donc comme variable d'étude le logarithme décimal de la masse des mottes, $\log \mathrm{M}_{\mathrm{m}}$, ce qui permettra de caractériser chaque distribution par une moyenne et un écart type.

La figure 4a présente l'évolution au cours du dessèchement de $\log \mathrm{M}_{\mathrm{m}}$ (moyenne, et intervalle de confiance 95 p. 100), pour les couches supérieures et inférieures.

Si la dispersion est un peu différente selon les couches (plus importante pour les couches supérieures), l'évolution globale de $\log \mathbf{M}_{\mathrm{m}}$ est identique sur tout le profil : dans une première phase de dessèchement (jusqu'à une humidité pondérale de 23 p. 100) la masse des mottes diminue, passant en moyenne de $250 \mathrm{~g}$ à $150 \mathrm{~g}$. Puis elle augmente régulièrement pour atteindre, en fin de dessèchement, une moyenne de $1500 \mathrm{~g}$ environ.

Ces importantes variations de la masse humide des mottes correspondent à de fortes variations de leur volume, modifiant ainsi la nature du réseau de fissures isolant ces mottes. D'un point de vue strictement qualitatif, on peut donc déjà admettre une évolution en 2 temps du réseau fissural au cours du dessèchement.
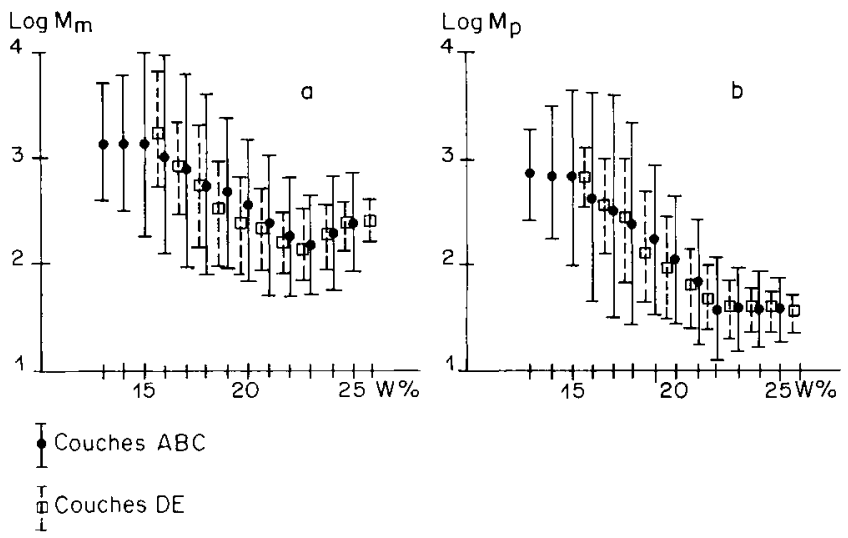

Figure 4

Variation du logarithme de la masse des éléments structuraux avec l'humidité pondérale $W$; moyennes et intervalles de confiance 95 p. 100. a) Masse des mottes ; b) masse des peds.

Logarithm of the mass of the structural units versus gravimetric water content $W$; means and $95 \%$ confidence intervals. a) Mass of the clods ; b) mass of the peds. 


\section{Evolution de la masse des peds}

Pour les mêmes raisons que précédemment, les 5 couches de sol ont été regroupées en couches supéieures et couches inférieures, et la variable d'étude est .e logarithme décimal de la masse des peds, $\log M_{p}$. Son évolution avec l'humidité pondérale est représentée sur la figure $4 \mathrm{~b}$.

Ici encore, si la dispersion des résultats différencie les couches, l'évolution moyenne est identique sur tout le profil. Nous retrouvons 2 phases de dessèchement, séparées par la même humidité que précédemment ; dans la $1^{\text {re }}$ phase, la masse des peds ne varie pas, restant en moyenne de l'ordre de $40 \mathrm{~g}$; puis elle augmente rapidement pour atteindre finalement 700 à $800 \mathrm{~g}$. On assiste donc ici aussi à d'importantes modifications du volume des peds, qui se répercuteront sur la structure du réseau de fissures intramottes dont l'évolution au cours du dessèchement semble s'opérer en 2 étapes.

\section{DISCUSSION}

Les méthodes utilisées caractérisent 2 types d'objets : d'une part les fissures (par mesure d'un indice des vides fissuraux), d'autre part les éléments de sol individualisés par ces fissures (mottes ou peds). Dans ce sol où les fissures forment un maillage fermé, mottes et peds constituent le complémentaire des fissures.

Or, quels que soient l'objet mesuré et le mode de dessèchement imposé (évaporation à l'air libre ou prélèvement racinaire), on observe une étroite similitude entre les résultats obtenus par ces méthodes : au cours d'un dessèchement continu, alors que la masse volumique des agrégats et des mottes augmente régulièrement, la fissuration du sol apparaît s'effectuer en 2 étapes, et le passage de l'une à l'autre intervient à une teneur en eau précise.

\section{A. Première phase de la fissuration : la fragmenta- tion}

- A saturation, aucune fissure n'est décelable à l'observation. Les faibles dispersions de la masse des mottes et de celle des peds indiquent cependant la persistance, dans un sol qui semble être à structure continue, de discontinuités mécaniques permettant d'individualiser des éléments structuraux d'environ $250 \mathrm{~g}$, eux-mêmes constitués d'éléments structuraux d'environ $40 \mathrm{~g}$.

- Entre la saturation et une humidité pondérale d'environ 23 p. 100 , le retrait provoque la création d'une porosité entre les mottes. Cette porosité s'organise en un réseau serré de fissures fines: elles sont décelables au sein des monolithes de sol, et elles individualisent des mottes de plus en plus petites. La fissuration au cours de cette phase occasionne donc une fragmentation du matériau.

L'absence de variation de la taille des peds durant cette phase semble indiquer la présence d'un maillage élémentaire au sein des mottes, correspondant à des zones de plus faible résistance. Dans le sol saturé, ce maillage ne se révèle qu'au moment de la chute des mottes. Lorsque le sol se dessèche, ces zones peuvent s'élargir en fissures fines, qui se révèlent alors dès le prélèvement de la motte : la taille de la motte se rapproche ainsi de celle du ped.

\section{B. Deuxième phase de la fissuration : la prise en masse}

Lorsque l'humidité pondérale est inférieure à 23 p. 100 , le retrait continue d'accroître le volume des vides externes aux mottes. Or, dans cette phase de dessèchement, les observations et mesures sur monolithes montrent une diminution du volume fissural à l'intérieur du cylindre. Ce phénomène, que nous avons dénommé prise en masse, résulte d'une augmentation du volume de sol sur lequel s'exerce le retrait : des fissures fines peuvent ainsi se fermer au sein des blocs pris en masse (ici, le monolithe) tandis que les fissures résiduelles sont de plus en plus espacées.

La taille des mottes et des peds, dont les masses sont respectivement multipliées par 10 et par 20 lorsque l'humidité passe de 23 à 16 p. 100, rend bien compte de cette prise en masse : il y a à la fois augmentation de l'écartement des fissures intermottes (car la taille des mottes augmente), et diminution de la porosité fissurale intramotte (car la taille des peds augmente plus vite que celle des mottes). Bénéficiant simultanément de l'accroissement du volume fissural total et de la fermeture des fissures intramottes, les fissures intermottes s'élargissent au cours de cette phase : un réseau lâche de fissures larges se substitue ainsi au réseau serré de fissures fines.

En conséquence à cette prise en masse, on observe donc un accroissement relatif de la taille du ped par rapport à celle de la motte : ceci peut être constaté sur la distribution de la masse des différents éléments issus d'une motte, ou, plus simplement, sur le rapport $\mathrm{M}_{\mathrm{p}} / \mathrm{M}_{\mathrm{m}}$. La figure 5 représente, pour chaque classe d'humidité, la moyenne et l'intervalle de confiance

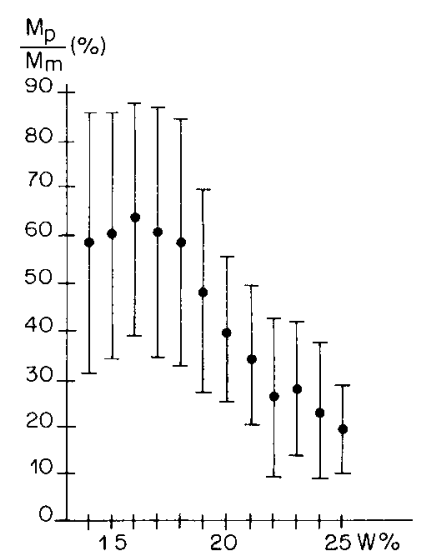

Figure 5

Variation du rapport masse des peds/masse des mottes avec l'humidité pondérale $W$; moyennes et intervalles de confiance 95 p. 100. Mass ratio versus gravimetric water content $W$; means and $95 \%$ confidence intervals. 
95 p. 100 de ce rapport (toutes couches de sol confondues). Il apparaît que, pour des humidités inférieures ou égales à 18 p. 100 , la masse du ped constitue en moyenne plus de la moitié de la masse de la motte dont il est issu : on peut considérer que, pour ces faibles humidités, la chute de la motte provoque une rupture mécanique à l'emplacement du choc, émettant des « éclats » et non plus des éléments structuraux. La motte peut alors être assimilée à un ped.

\section{Schéma global d'évolution structurale du sol}

\section{Conséquences structurales de l'affaissement}

Sur ce même type de sol, des mesures du retrait vertical (HALLAIRE, 1987) avaient fait apparaître un processus se déroulant aussi en 2 temps : jusqu'à une humidité de 23 p. 100 environ, l'affaissement des couches de sol est moindre que l'affaissement cumulé des agrégats qui les composent (ceux-ci étant supposés avoir un retrait isotrope) ; en dessous de cette humidité, ce retard tend à s'atténuer, pour s'annuler en fin de dessèchement. Pour expliquer ce « retard à l'affaissement " des couches par rapport à leurs agrégats, on peut imaginer plusieurs scénarios :

- ce retard à l'affaissement pourrait être expliqué par l'absence de retrait dans une première phase du dessèchement : celle-ci serait due à la vidange de pores non déformables internes aux agrégats; le retrait n'interviendrait progressivement que dans une seconde phase,

- il pourrait aussi être expliqué par une anisotropie du retrait des agrégats : si ceux-ci se déforment au cours du retrait, l'épaisseur de la couche sera différente de l'épaisseur calculée selon l'hypothèse d'un retrait isotrope des agrégats,

- il pourrait enfin être expliqué par la création, au cours du retrait, d'une fissuration comportant une composante horizontale, s'opposant à l'empilement parfait des agrégats ; même si ceux-ci ont un retrait isotrope, l'épaisseur de la couche sera supérieure à l'épaisseur cumulée de ses agrégats.

Ces 3 scénarios aboutissent à des états structuraux différents :

- dans le premier, il n'y aura pas de fissuration tant que la vidange des pores sera seule responsable du dessèchement (absence de retrait),

- dans le second, la fissuration sera exclusivement verticale, car les agrégats restent parfaitement empilés les uns au-dessus des autres,

- dans le troisième, la fissuration sera multidirectionnelle, car l'une de ses composantes, horizontale, s'opposera à l'empilement parfait des agrégats.

Quel que soit le mécanisme mis en cause, on voit que l'affaissement n'est pas indépendant de la fissuration. Le choix du plus probable de ces scénarios doit donc prendre en compte les données que nous avons recueillies concernant l'évolution des réseaux de fissures.

\section{Relations entre la fissuration et l'affaissement}

Il nous semble d'autant plus justifié de relier ces 2 phénomènes qu'ils présentent un comportement analogue : pour chacun d'eux on a pu décrire 2 étapes au cours du dessèchement, ces 2 étapes étant séparées par la même valeur d'humidité.

$\mathrm{Au}$ cours de la première phase du retrait, nous avons vu se développer une microfissuration intense. Si l'on extrapole ce phénomène de fragmentation dans les 3 dimensions, il est parfaitement logique de considérer que ces fissures, nombreuses et fines, s'opposent à l'emboîtement des agrégats selon l'axe vertical. Cela correspond à la composante horizontale de la fissuration, que nous avons évoquée dans le troisième scénario : elle provoque un retard à l'affaissement d'autant plus important que la microfissuration est intense, c'est-à-dire que l'on se rapproche de l'humidité de 23 p. 100. Durant cette phase de fragmentation, le réseau de fissures est donc non seulement dense et fin, mais il est également multidirectionnel.

La seconde phase du retrait, la prise en masse, provoque une redistribution des espaces fissuraux permettant un meilleur emboîtement des agrégats. Il en résulte, dans le plan horizontal, une fermeture d'une partie des fissures au profit d'un réseau macrofissural de moins en moins dense. Selon l'axe vertical, la prise en masse se traduira par la disparition progressive de leur composante horizontale, au profit d'un affaissement plus rapide de la couche de sol : le retard à l'affaissement diminue à partir de 23 p. 100. Les fissures, plus larges et plus espacées que dans la première phase, acquerront ainsi une orientation verticale préférentielle.

\section{CONCLUSION}

Au cours du dessèchement, le retrait provoque une augmentation continue du volume fissural total. Or, sur le sol étudié, cette augmentation ne se traduit pas par la création continue de nouvelles fissures. Les résultats montrent en effet clairement 2 phases de fissuration ; la première phase accroît l'épaisseur des fissures dont le maillage semble déterminé dès la saturation ; il en résulte un réseau dense de fissures fines. La seconde phase referme une partie des fissures, en continuant d'accroître l'épaisseur des fissures résiduelles ; il en résulte un réseau plus lâche de fissures larges.

Le rapprochement des méthodes utilisées souligne l'intérêt d'une caractérisation structurale de ce type de sol : l'organisation des fissures en réseau nous permet d'appréhender leur maillage en étudiant les volumes de sol qu'elles définissent. Cette approche ne supprime cependant pas toutes les difficultés intervenant dans la détermination de ces volumes, notamment des mottes pour les humidités élevées et des peds pour les faibles humidités.

Le mécanisme des processus mis en évidence - fragmentation et prise en masse - reste à déterminer : son étude nécessiterait une analyse plus fine de la répartition des potentiels hydriques au sein de la motte, et des contraintes qui en résultent, notamment pour l'humidité de 23 p. 100 . En l'absence de données plus précises, on ne peut dans l'état actuel que proposer des hypothèses : n'y a-t-il pas, pour cette teneur en eau particulière, changement de l'organisation sans changement de la valeur du potentiel de l'eau ? Cette humi- 
dité correspond-elle à une valeur seuil dans le comportement rhéologique du matériau?

Si ces questions restent actuellement sans réponses, les résultats présentés ici montrent que les conséquences macroscopiques du retrait sur l'organisation structurale du sol sont suffisamment complexes, pour qu'une description morphologique précise de ce phé- nomène apparaisse prioritaire à toute tentative de modélisation ; c'est pourquoi, après avoir décrit ici l'évolution des réseaux de fissures de manière qualitative, nous nous attacherons dans une seconde partie à en quantifier les composantes.

Reçu le 15 janvier 1987. Accepté le 24 novembre 1987.

\section{RÉFÉRENCES BIBLIOGRAPHIQUES}

Berndt R. D., Coughlan K. J., 1976. Bulk density changes in a cracking clay soil. Aust. J. Appl. Sci., 4, 244-259.

Brewer R., 1964. Fabric and mineral analysis of soils. 1 vol., John Wiley and Sons, New York, $470 \mathrm{p}$.

Bruand A., 1985. Contribution à l'étude de la dynamique de l'organisation de matériaux gonflants. Thèse de Spéc. Paris III, 225 p.

Cabidoche Y. M., Jaillard B., Ney B., 1986. Dynamique de l'eau dans les vertisols sur calcaires récifaux, p. 449-478. In Sol et Eau, Actes du Séminaire de La Havane, 8-20 avril 1985, ORSTOM Paris, $767 \mathrm{p}$.

De Vos J. H., Virgo K. J., 1969. Soil structure in vertisols of the Blue Nile clay plains, Sudan. J. Soil Sci., 20 (1), 189-206.

Edelman C. M., Brinkman R., 1962. Physiography of gilgaï soils. Soil Sci., 94 (6), 366-370.

Fiès J. C., Stengel P., 1981 $a$. Densité texturale de sols naturels. I. Méthode de mesure. Agronomie, 1 (8), 651-658.

Fiès J. C., Stengel P., 1981b. Densité texturale de sols naturels. II. Eléments d'interprétation. Agronomie, 1 (8), 659-666.

Fox W. E., 1964. A study of bulk density and water in a swelling soil. Soil Sci, 98 (5), 307-316.

Franzmeier D. P., Ross S. J., 1968. Soil swelling : laboratory measurement and relation to other soil properties. Soil Sci. Soc. Am. Proc., 32, 573-577.

Haines W. B., 1923. The volume changes associated with variations of water content in soil. J. Agric. Sci., 13, 293-310.

Hallaire V., 1984. Evolution of crack networks during shrinkage of a clay soil, p. 49-54. In J. Bouma \& P. A. C. Raats, ed. Proceedings of the ISSS symposium on water and solute movement in heavy clay soils, ILRI publication 37, Wageningen, $363 \mathrm{p}$.
Hallaire V., 1987. Le retrait vertical d'un sol argileux au cours du dessèchement. Mesures de l'affaissement et conséquences structurales. Agronomie, 7 (8), 631-637.

Holmes J. W., 1955. Water sorption and swelling of clay blocks. $J$. Soil Sci., 6, 200-208.

Monnier G., Stengel P., Fiès J. C., 1973. Une méthode de mesure de la densité apparente de petits agglomérats terreux. Application à l'analyse des systèmes de porosité du sol. Ann. agron., 24 (5), 533545 .

Rijniersce K., 1984. Crack formation in newly reclaimed sediments in the IJsselmeer polders, p. 59-62. In J. Bouma \& P. A. C. Raats, ed. Proceedings of the ISSS symposium on water and solute movement in heavy clay soils, ILRI publication 37, Wageningen, $363 \mathrm{p}$.

Stengel P., 1979. Utilisation de l'analyse des systèmes de porosité pour la caractérisation de l'état physique du sol in situ. Ann. agron., 30 (1), 27-51.

Tessier D., Pédro G., 1980. Sur les propriétés de gonflement des argiles dans les sols. C. R. Acad. Sci., Paris, sér. D, 291 (5), 461-464.

Tessier D., 1984. Etude expérimentale de l'organisation des minéraux argileux. Thèse Doci. ès Sci. Paris VII, I.N.R.A., 361 p.

Virgo K. J., 1981. Observations of cracking in Somali vertisols. Soil Sci., 131 (1), 60-61.

White E. M., 1962. Volume changes in some clay soils. Soil Sci., 94, 168-172.

Yule D. F., Ritchie J. T., 1980a. Soil shrinkage relationships of Texas vertisols. I. Small cores. Soil Sci. Soc. Am. J., 44 (6), 12851291.

Yule D. F., Ritchie J. T., 1980b. Soil shrinkage relationships of Texas vertisols. II. Large cores. Soil Sci. Soc. Am. J., 44 (6), 12911295. 